\title{
Miniature near infrared spectroscopy spectrometer and information and communication technologies to guarantee the integrity of the EU high added-value "acorn Iberian pig ham" (IP)
}

\section{Ana Garrido-Varo, Cecilia Riccioli, Tom Fearn, E. De Pedro, Dolores Pérez-Marín}

Ana Garrido-Varo, Cecilia Riccioli, Tom Fearn, E. De Pedro, Dolores Pérez-Marín, "Miniature near infrared spectroscopy spectrometer and information and communication technologies to guarantee the integrity of the EU high added-value "acorn Iberian pig ham" (IP)," Proc. SPIE 10665, Sensing for Agriculture and Food Quality and Safety X, 106650M (7 June 2018); doi: 10.1117/12.2299641

Event: SPIE Commercial + Scientific Sensing and Imaging, 2018, Orlando, Florida, United States 


\title{
Miniature near infrared spectroscopy spectrometer and information and communication technologies to guarantee the integrity of the $\mathbf{E U}$ high added-value "acorn Iberian pig ham” (IP)
}

\author{
Ana Garrido-Varo ${ }^{1 *}$, Cecilia Riccioli ${ }^{1}$, Tom Fearn ${ }^{2}$, E. De Pedro ${ }^{1}$ and Dolores Pérez- Marín ${ }^{1}$ \\ ${ }^{1}$ Non- Destructive Spectral Sensors UNIT. Faculty of Agricultural and Forestry Engineering, \\ University of Córdoba, Córdoba, Spain.*pa1gavaa@uco.es \\ ${ }^{2}$ Department of Statistical Science, University College London, UK.
}

\begin{abstract}
This research is framed within FoodIntegrity, EU sponsored project (7th FP). The main goal of the research to be done is to provide industrials, producers and consumers with a methodology based in low-cost, portable and miniature NIRS sensors and information and communication technologies for process control and voluntary labelling, to guarantee the integrity of the EU high added-value as the "acorn Iberian pig ham". The present study is focussed in transferring a database (470 samples) of IP tissue - analysed in a FOSS-NIRSystems 6500 (FNS6500) spectrometer, during the seasons 2009-2011 - to a portable/miniature instrument MicroNIR-Onsite, VIAVI (MN1700). A set of 30 samples of adipose tissue was taken from a slaughterhouse during 2015-2016, being analysed in parallel in the satellite (FNS 6500) and master (MN 1700) instruments. Latter on, they were divided in two sets: $\mathrm{N}=10$ for building the standardization matrices and $\mathrm{N}=20$ for the validation of the cloning procedure. The algorithm Piece-Wise Direct Standardization (PDS) was applied. The best standardisation matrix was applied to the library of 470 samples taken in the FNS 6500, enabling an excellent fitting between both instruments, as shown the RMSCs statistic calculated in the satellite before and after the standardization and in the master - 108457 vs 22519 vs $17646 \mu \log 1 / \mathrm{R}$ - and the GH distance before and after standardisation between both instruments 437.41 vs 2.06 .
\end{abstract}

Keywords: instruments standardization, spectral matching, on-site NIR analysis, Acorn Iberian pig ham

\section{INTRODUCTION}

Acorn Iberian ham ("Jamón Ibérico de Bellota”) is one of the most expensive luxury food products produced in Europe. Since January 2014, a new system of labelling for Iberian ham products came into force in Spain aiming at avoiding mislabelling and frauds in the market, where prices for a cured leg of Iberian ham range from hundreds to thousands of euros. Currently, the official quality-control systems used for determining the feeding regime of Iberian pigs are just based on on-farm inspection, while in the past also laboratory analysis of the fatty-acid composition of melted subcutaneous fat using gas chromatography (GC) was used. Nevertheless, GC is still used at industry level for production self-control and producer payment.

However, both methods are costly and timeconsuming, and only provide information on batches of animals rather than individual pigs, an important consideration where the product, as in this case, reaches high market prices. Usually, in the slaughterhouse, one fat sample per animal is taken for each batch (each batch being made up of between 30 and 80 animals). All these samples are then melted together and a single GC analysis is performed. The fatty acid profile of individual pigs may differ greatly from the batch average. This, together with the costly and laborious nature of the

Sensing for Agriculture and Food Quality and Safety X, edited by Moon S. Kim, Kuanglin Chao,

Bryan A. Chin, Byoung-Kwan Cho, Proc. of SPIE Vol. 10665, 106650M · C 2018 SPIE

CCC code: $0277-786 \mathrm{X} / 18 / \$ 18 \cdot$ doi: $10.1117 / 12.2299641$ 
procedure involved and the inability to be implemented on-line in the plant are the main disadvantages of the traditional control system. Rigorous instantaneous and on-line control procedures are therefore required to ensure the traceability, authentication and quality of Iberian pork products, in order to guarantee product quality and homogeneity for the industry, and satisfy consumer expectations.

Over the past 20 years, several papers (De Pedro et al., 1992; Fearn et al., 2010; García et al. 1998; Garrido et al. 2004, 2012; Garrido and De Pedro, 2007; Hervás et al. 1994; Pérez-Marín et al., 2007, 2008,2009, 2012a,b; Zamora 2013; Zamora et al., 2012a, 2013) have confirmed the suitability of the NIR technology for the for the rapid, non-destructive, on-site and cost-effective characterization of a great diversity of agri-food products, and in particular Iberian ham.

The main objective of this paper is to optimize a portable miniature spectrometer for the on-site analysis of individual pork carcasses and transfer existing NIRS data libraries from a monochromator instrument used for at-line analysis, to the portable instrument to be used "on-site".

To reach this objective, a methodology based in the concept of "spectral matching” (Fearn, 2001; García et al. 2001)was used - as opposite to the traditional way used by most NIR vendors- who correct "provissionaly" the problem by calculating bias and slope variations for every set of samples analysed in the individual host instrument and incorporate these corrections into the calibration. From the scientific point of view, a good deal of skepticism existing concerning this bias and slope approach to manage the many variation sources existing from one NIR instrument to another.

The idea behind "spectral matching", used in our study, is to make different instruments as much similar as possible, so they can use the same spectral libraries with minimal loss of precision and accuracy. The procedure to move a database from one device to another different requires two basic steps: appropiate selection of a set of representative samples (in our case Iberian pig adipose tissue) - which must be scanned in both instruments- and the evaluation of different mathematical corrections (cloning/standardization algorithms).

\section{MATERIAL AND METHODS}

\subsection{Instrumentation}

Three NIR instruments have been used:

- A FOSS NIRSystems 6500 (FNS6500) monochromator spectrometer (FOSS-NIRSystems Inc., Sylver Spring, MD, USA), equipped with an interactance-reflectance fiber optic and covering the spectral range $400-2500 \mathrm{~nm}$, with a spectral interval of $2 \mathrm{~nm}$. The instrument was connected to desktop computer provided with the WINISI 1.5 software (Infrasoft International, USA). According to previous research, the regions between 400-450 nm and between 2208-2498 nm were discarded; the latter region is particularly associated with a poor signal/noise ratio, due to the interference-taking place in fibre-optic light transmission (García et al. 1998; Pérez-marín et al. 2009; Zamora et al. 2012).

- Two portable/miniature instruments namely MicroNIR Onsite Lite (MN1700) and MicroNIR Pro 2200 ES (MN2200) both commercialized by Viavi Solutions Inc. (formerly JDSU Corporation, Santa Rosa, CA). The MN1700 covers the range 900-1700 with an approximately spectral interval of $6.2 \mathrm{~nm}$. The MN1700 is the most updated version of Micronir available from VIAVI. The MN2200 covers the range 1100-2200 nm, with a spectral interval of about $8.1 \mathrm{~nm}$, but the optical design is an old version not improved. Table 1 shows the main features of the three instruments. 
Table 1. Main characteristics of the used instruments

\begin{tabular}{llll}
\hline & FNS6500 & MN1700 & MN2000 \\
\hline Useful Wavelength range & $400-2200$ & $932-1676$ & $1207-2104$ \\
\hline Spectral repeatability $(\mu \log 1 / \mathrm{R})$ & 250 & 800 & 800 \\
\hline Spectral resolution & $2 \mathrm{~nm}$ & $6.19 \mathrm{~nm}$ & $8.09 \mathrm{~nm}$ \\
\hline Measuring time & $30 \mathrm{~s}$ & $3-5 \mathrm{~s}$ & $3-5 \mathrm{~s}$ \\
\hline Integration time & $>1875 \mathrm{~ms}$ & $>10 \mu \mathrm{s}$ & $>10 \mu \mathrm{s}$ \\
\hline Dimensions & $25 \times 35 \times 29 \mathrm{~cm}$ & $14.6 \times 4.45 \mathrm{~cm}$ & $4.5 \times 4.2 \mathrm{~cm}$ \\
\hline Weight & $25 \mathrm{Kg}$ & $<200 \mathrm{~g}$ & $64 \mathrm{~g}$ \\
\hline
\end{tabular}

Despite the existing MN2200 has not suffered the same refinement in optics and electronics than the MN1700, it has been also considered in the research done taking into account that the intended applications could demand longer wavelengths. Both spectrometers were connected to a rugged 7'' Windows 8.1 tablet provided with the software version 2.1 (Viavi Solutions Inc.).

\subsection{Cloning/standardization}

\subsubsection{Selection of samples}

The Universidad de Córdoba (UCO) had available a previous database of Iberian pig spectra, made up of about 500 samples of adipose tissue - with a high variability in feeding regimes, fatty acids profile, breeds - analyzed in the FNS 6500 during the slaughtering periods of 2009-2011. Additionally, a separate set consisting of 66 "new" samples of subcutaneous adipose tissue were taken from a commercial slaughterhouse during the slaughter campaign of 2015-2016. (Figure 1) Samples obtained in the slaughterhouse were placed in appropriately tagged plastic bags and transported to the laboratory at the University of Córdoba, where they were stored at $-20^{\circ} \mathrm{C}$ until 24 hours before NIRS analysis. A subsample of each adipose tissue sample was melted in a microwave oven following De Pedro et al. (2013). Once melted, the fatty acid composition of each sample was determined by gas chromatography (GC).

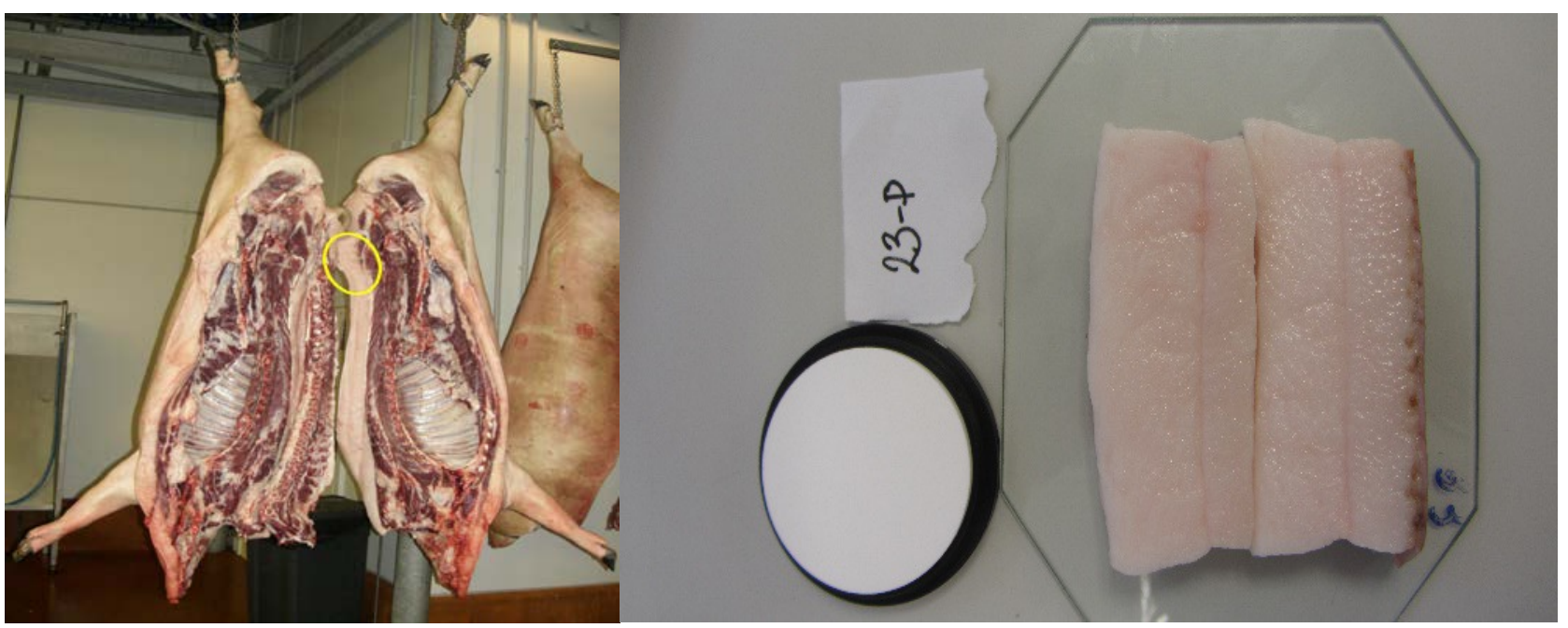

Figure 1. Samples taken from the tail insertion area in the coxal region (left) and samples ready for NIR analysis (right).

All the cloning/standardization process was undertaken at UCO laboratory, where the three instruments were located, in order to avoid any change in the samples during the process. The 66 "new" samples were selected having into account information provided by collaborator producers and FAs were determined by GC at "Laboratorio Agroalimentario de 
Córdoba” (local laboratory). Based on previous research done by UCO (Zamora et al. 2012; Zamora et al. 2012), it was decided to use ten samples for standardization and twenty samples for the validation of the process. They were selected on the basis of their Mahalanobis distance (GH) from the center of the population using the algorithm CENTER (Shenk, J.S., \& Westerhaus, 1991). The remaining thirty-three of the 66 samples were included into the original database in order to cover the natural variability which is expected to encounter in the IP production rearing systems in the period 20142015 (i.e., more or less available natural resources, changes in complementary feed etc.) as compared to the samples of the years 2009-2011.

\subsubsection{Design of a protocol for recording spectra of IP adipose tissue in parallel in the three instruments}

The protocol is based in the experience gained from cloning of the FNS6500 and other portable instruments using adipose IP tissue (García et al. 2001). Spectral data were collected under strictly controlled conditions (temperature maintained around $22^{\circ} \mathrm{C} \pm 0.5^{\circ} \mathrm{C}$ and relative humidity were unchanged at around $50 \% \pm 5 \%$ ). It is important to be noticed that controlled conditions are only a key aspect for the standardization process and not for the routine analysis.

Before starting the scanning of the standardization and validation samples in each instrument, the FNS6500 instrument working conditions were evaluated by means of the diagnostic and check-cell tests proposed by Shenk (1991). For the MN instruments, the protocol recommended by VIAVI includes a quality test in order to check the spectroscopic noise, wavelength uncertainty and photometric linearity.

Once performance of the instrument has been evaluated, the next step in the cloning/standardization protocol is to scan the samples in the master and satellite instrument. All the 66 samples were analyzed in three NIRS instruments (Figure 2).
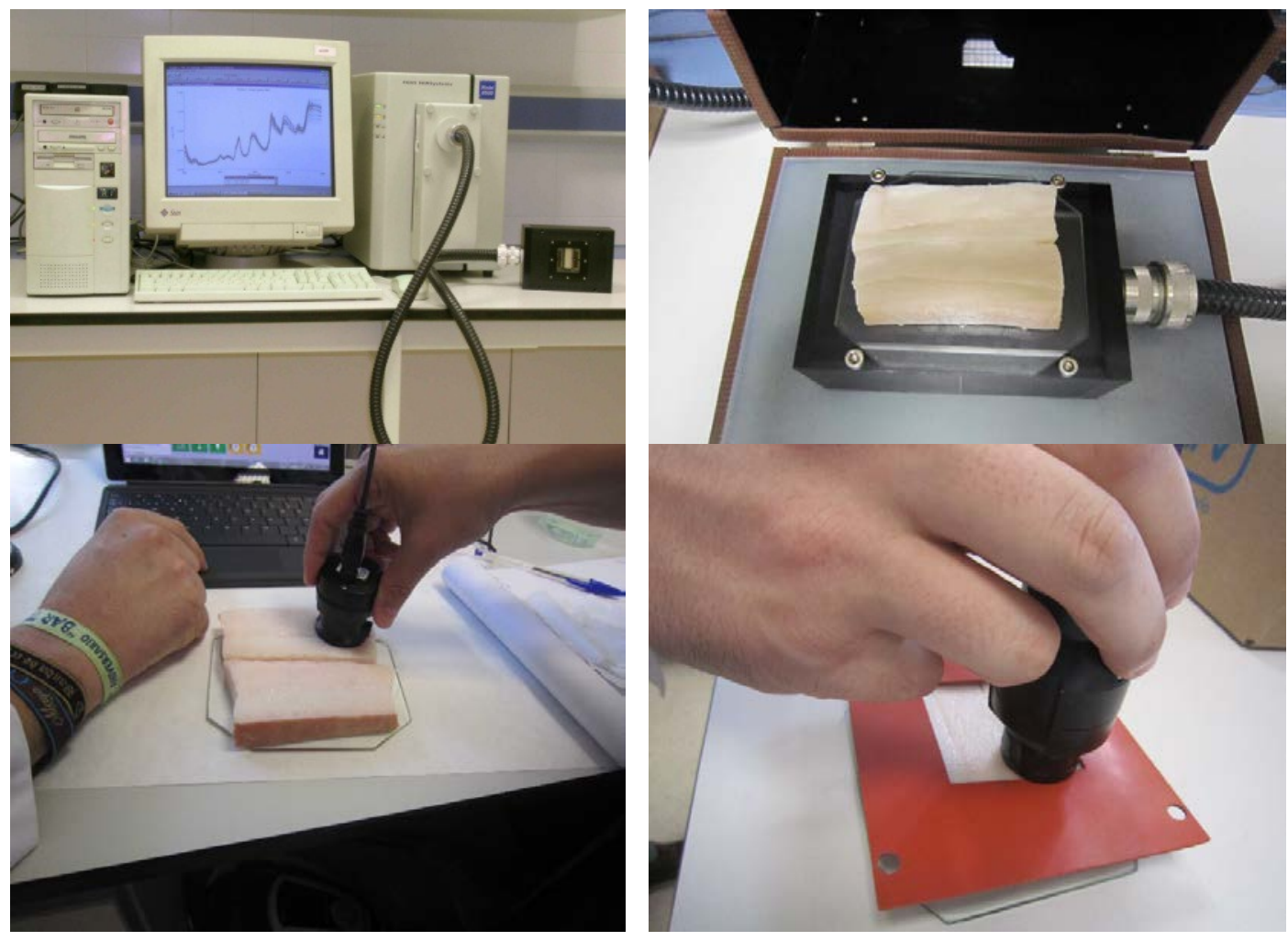

Figure 2. Samples of pig adipose tissue analyzed with the FNS6500 (top) and MN (bottom).

Two and four replicates per sample were taken in the FNS6500 and the MNs, respectively. As previously mentioned, the FNS 6500 spectra were recorded using the WINISI software and those from the MNs were collected using MicroNIR Pro spectrometer software version 2.1 (Viavi Solutions Inc.) and exported to .csv format for further processing. 


\subsubsection{Standardization/cloning algorithms}

A total of eight standardization matrices were evaluated, combining the different sample groups ( $\mathrm{N}=1$ and $\mathrm{N}=10$ samples) and the four cloning algorithms (direct standardization (SD), piecewise direct standardization (PDS), double windows PDS (DWPDS) and spectral difference by wavelengths (SDW)).

Since the three instruments work at different resolutions and over different spectral ranges, prior to the development of the standardization matrices, the spectra taken in the different instruments were adjusted using the interpolation function "spline" included in the MATLAB software. The standardization matrices were developed using the MATLAB R2016a software package (TheMathWorks, Natick, USA). The prediction algorithms were developed using WinISI version 1.50 software package (Infrasoft Internacional, Port Matilda, PA, USA).

The similarity of the spectra before and after standardization were quantified through visual graphical inspection and by using the following two statistics:

1) RMS (c), used to compare spectra for the same sample obtained on the two instruments, was calculated using the CONTRAST algorithm included in the WINISI program.

$\operatorname{RMS}(\mathrm{c})=10^{6} \mathrm{x} \sqrt{\frac{\sum_{\mathrm{i}=1}^{\mathrm{n}} \mathrm{D}_{1}^{2}-\frac{\left[\sum_{\mathrm{i}=1}^{\mathrm{n}}\left(y_{\mathrm{i} \text { master }}-y_{i} \text { satellite }\right)\right]^{2}}{\mathrm{n}}}{\mathrm{n}-1}}$

where $\mathrm{Di}=$ yi master-yi satellite are the $\log (1 / \mathrm{R})$ values of two spectra of a single sample scanned in master and satellite instruments at a given wavelength.

2) Data Difference (DD) defined as:

$\mathrm{DD}=\sqrt{\frac{\sum\left(y_{\text {imaster }}-y_{i} \text { satellite }\right)^{2}}{\sum \mathrm{y}_{\text {master }}^{2}}}$

3) Mahalanobis distance. Values were calculated for the statistic global H (GH), i.e. the distance of a given sample from the center of the population; limits were set at 3 (3 standard deviations from the mean) (Shenk, J.S., \& Westerhaus, 1991).

\section{RESULTS AND DISCUSSION}

\subsection{Optimization of spectral range and resolution}

Optimizing a portable handheld device for the on-site application to Iberian pork carcasses at the slaughterhouse is no easy task from the scientific point of view. Evaluation of the specifications provided by manufacturers is critical, and also the assessment of performance parameters such as noise, wavelength accuracy and detector response. Furthermore, determination of the final useful wavelength range is of paramount importance. The principal goal of Task 19.1 was the selection of the optimal spectral range and this could be possible by the comparison of MN1700 and MN2500 performances in terms of spectral repeatability, spectral noise and prediction ability.

Once all the tests were successfully accomplished, spectral repeatability from both instruments was compared.

The difference in spectral range between instruments can be observed in Figure 3 where the average spectra of the 66 "new" samples analyzed with the three instruments has been represented. 


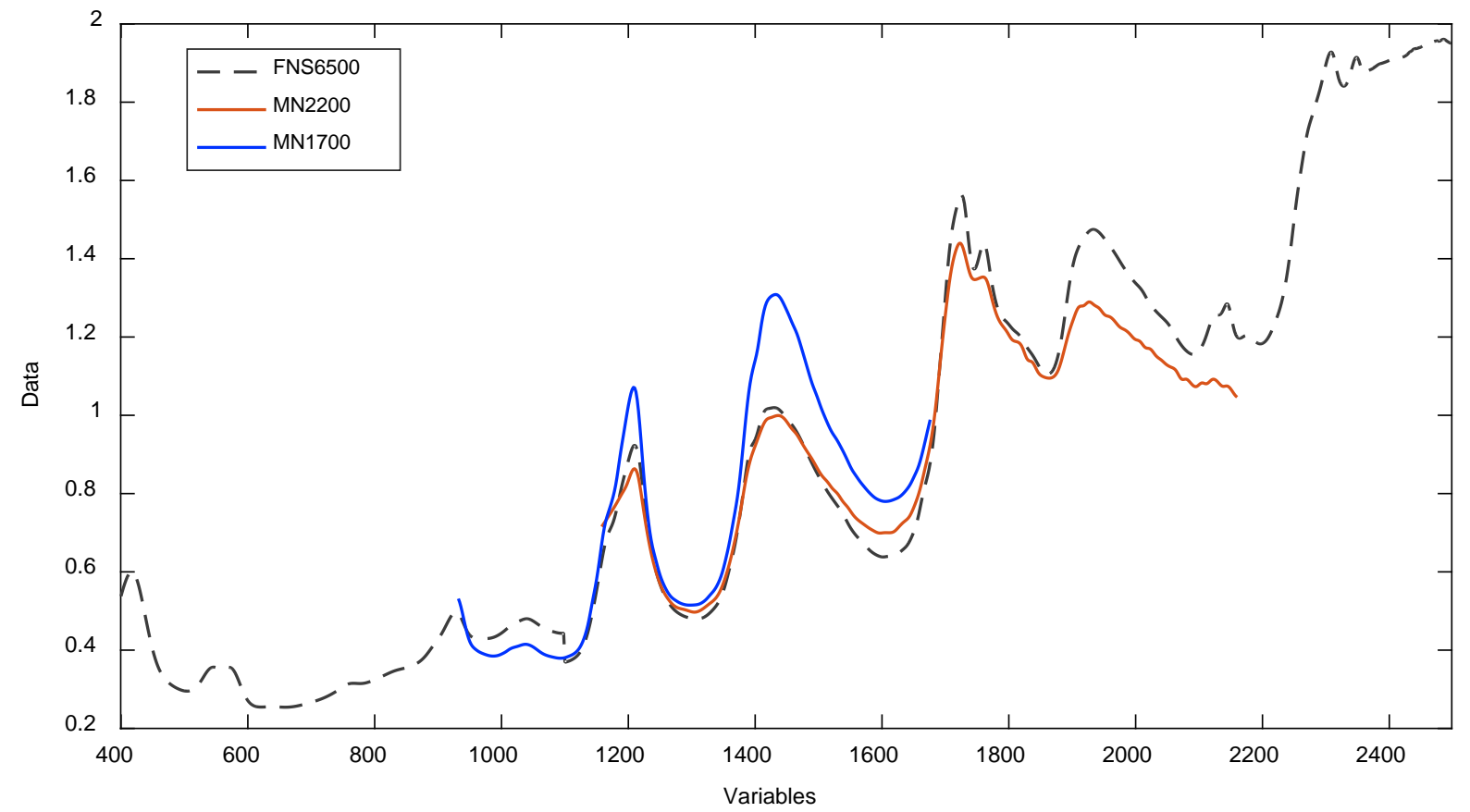

Figure 3. Average spectra for ( $N=66$ ) IP samples measured with FNS6500 and MN devices.

Since the FNS6500, MN1700 and MN2200 spectrometers also differ in spectral wavelength interval step, a cubic spline interpolation (De Boor 1978) was used. Two different strategies were compared:

1) trim the FSN6500 range and resolution to the MN1700 (932-1676, $\sim 6 \mathrm{~nm}$ ) and to the MN2200 (1158-2104, $~ 8 \mathrm{~nm})$ ones, to enable precise adaptation to MN1700 and MN2200 devices respectively

2) trim aqui seria interpolate no? the MN1700 and MN2200 spectrometers resolution to the FSN6500 (2 nm) one, once cut trimmed the spectra to the MN1700 and MN2200 ranges respectively.

Figure 3 shows the average spectra for adipose tissue samples $(\mathrm{N}=66)$ obtained using the three instruments and with different resolutions. 

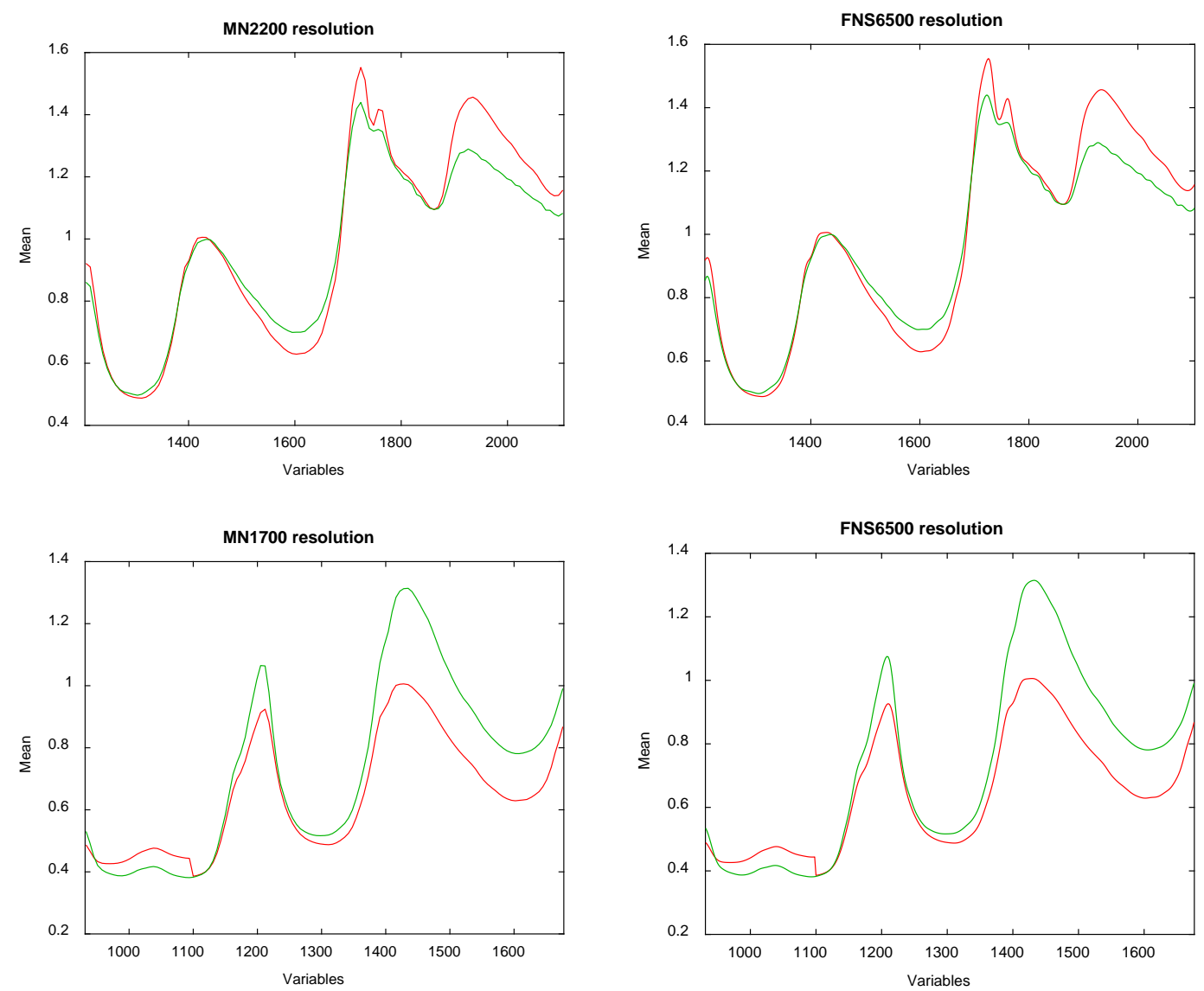

Figure 4. Comparison between the mean spectrum analyzed with FNS6500 (red) and the mean spectrum analyzed with the MN devices (green) at MN resolution (left) and FSN6500 resolution (right) for both MN instruments ranges.

MN instruments yielded similar spectral patterns to those achieved in FNS6500 (absorption peaks aligned on the horizontal axis). However, evident differences were found between spectra with regard to absorbance, attributable to differences between instruments. The similarity between replicates (two in the case of FNS6500 and four in the case of MN1700 and MN2500) was studied by calculating the RMS (Table 1).

Table 1. RMS average values for the three instruments (FNS6500, MN1700 and MN2500) at different spectral resolutions (MN2200, MN1700 and FNS6500)

\begin{tabular}{l|l|l|l|l|l|l|l|l}
\hline Instrument & FNS6500 & FNS6500 & FNS6500 & FNS6500 & MN1700 & MN1700 & MN2200 & MN2200 \\
\hline $\begin{array}{l}\text { Resolution } \\
(\mathrm{nm})\end{array}$ & 2 & 2 & $\sim 8$ & $\sim 6$ & 2 & $\sim 6$ & 2 & $\sim 8$ \\
\hline $\begin{array}{l}\text { Range } \\
(\mathrm{nm})\end{array}$ & $1158-2104$ & $932-1676$ & $1158-2104$ & $932-1676$ & $1158-2104$ & $932-1676$ & $1158-2104$ & $1158-2104$ \\
\hline $\begin{array}{l}\text { RMS } \\
(\mu \log 1 / \mathrm{R})\end{array}$ & 7.023 & 4.014 & 7.017 & 4.021 & 21.030 & 21.033 & 34.934 & 34.913 \\
\hline
\end{tabular}


The RMS values showed that MN instruments have, in general, lower spectral repeatability compared to the FNS6500. This is probably due to their different optic and electronic designs as well as to differences in the spectral resolutions (lower for the MN instruments compared to the FNS6500). No porque si te efijas a igualdad de resolución el FNS siempre tiene valores mas bajos Also, the differences in the size of the windows of analysis as well in the frequency of the reference material scanning can have influence in the spectral repeatability. The FNS6500 is a monochromator instrument, specifically designed for research purposes, and the MN instruments are both low-cost instruments, thus it is expected some loose in performance.

The MN2200 spectrometer has lower spectral repeatability compared to the MN1700, probably due to its not improved optic design and its lower spectral resolution ( 8 $\mathrm{nm}$ ) compared to MN1700 ( 6 nm). Regarding the spectral wavelength interval step, no significant variations were observed when using the higher resolution (FNS6500) compared to the lower one (MN devices). This result, coupled with the fact that MN2200 lacks some improvements such as an ergonomic handle compared to MN1700, prompted us to choose the latter instrument as the one to be used from then on. Therefore, only the MN1700 will be evaluated and optimized along the project.

In order to further evaluate the influence of spectral resolution and range differences, modified Partial Least Square Regression (M-PLS) models equations were constructed using the FNS6500 and the database of 500 samples over different resolutions: a non-constant step of around $6 \mathrm{~nm}$ corresponding to the MN1700 resolution and a step of $2 \mathrm{~nm}$ corresponding to the FNS6500. The results are shown in Table 2. The equations were developed with $\mathrm{N}=526$ samples and validated with a set of $\mathrm{N}=20$ samples.

Table 2. Comparative of the FA models developed in the FNS 6500 statistics depending on the wavelength intervals (or resolution).

\begin{tabular}{lllll}
\hline Parameter & Spectral resolution & SECV & SEP & $\mathrm{R}^{2} \mathrm{p}$ \\
\hline Palmitic (C16:0) & FNS6500 $(2 \mathrm{~nm})$ & 0.293 & 0.648 & 0.969 \\
\cline { 2 - 5 } & FNS6500 $(\sim 6 \mathrm{~nm})$ & 0.415 & 1.657 & 0.930 \\
\hline Stearic (C18:0) & FNS6500 $(2 \mathrm{~nm})$ & 0.230 & 0.727 & 0.922 \\
\cline { 2 - 5 } & FNS6500 $(\sim 6 \mathrm{~nm})$ & 0.295 & 0.753 & 0.916 \\
\hline Oleic (C18:1) & FNS6500 $(2 \mathrm{~nm})$ & 0.575 & 0.984 & 0.974 \\
\cline { 2 - 5 } & FNS6500 $(\sim 6 \mathrm{~nm})$ & 0.872 & 3.553 & 0.959 \\
\hline Linoleic (C18:2) & FNS6500(2 nm) & 0.235 & 0.447 & 0.792 \\
\cline { 2 - 5 } & FNS6500 $(\sim 6 \mathrm{~nm})$ & 0.291 & 0.719 & 0.404 \\
\hline
\end{tabular}

In Table 2 it can be observed that, with the exception of prediction for Stearic Acid, all the statistics are significantly worsened by the use of the lower resolution $(\sim 6 \mathrm{~nm})$. Regarding the prediction equation for the primary fatty acid (C18:1) the SEP is 3,6 times bigger than the SEP obtained using the higher resolution (2 $\mathrm{nm}$ ). The best results in terms of accuracy in Cross Validation (SECV) and validating with an external dataset (SEP) in this first stage were obtained analyzing intact adipose samples with an interval of $2 \mathrm{~nm}$ for all the fatty acids. This resolution was consequently selected as the one to be used henceforth. Results for $\mathrm{R}^{2} \mathrm{p}$ and SEP showed in Table 2 also represent the target statistics for the best models that could be obtained with the MN1700 instrument once the spectral library will be transferred from the FNS6500 to the MN1700 and models will be developed using 2nm resolution.

\subsection{Instrument cloning}

A key factor in the cloning process is the number of samples used when selecting a cloning algorithm (De Pedro et al. 2013; De Boor 1978). Here, 66 samples were analyzed in parallel and, as explained in section 2.2.3, two strategies were 
compared: the selection of ten samples using the CENTER algorithm and the selection of one sample. Both sets of samples were used to build the cloning matrices, while an amount of 36 samples were included into the model and the rest $(\mathrm{N}=20)$ were used as a cloning validation set for the evaluation of the different cloning algorithms. The use of one sample (with a spectrum similar to the average of the 66 samples) provided worst results in terms of RMS (c), DD and GH than the use of ten samples covering the spectral variation encountered in the full data set (results not shown here), so this latter strategy was employed for further analysis. After removing spectral outliers, an amount of 470 samples were subjected to standardization. Figure 5 show the average spectra for the cloning validation set obtained in the FSN6500 before and after standardization using four matching matrices corresponding to the four cloning methods, namely DS, PDS, DWPDS and SDW, compared to the average spectrum for the samples from the MN1700.
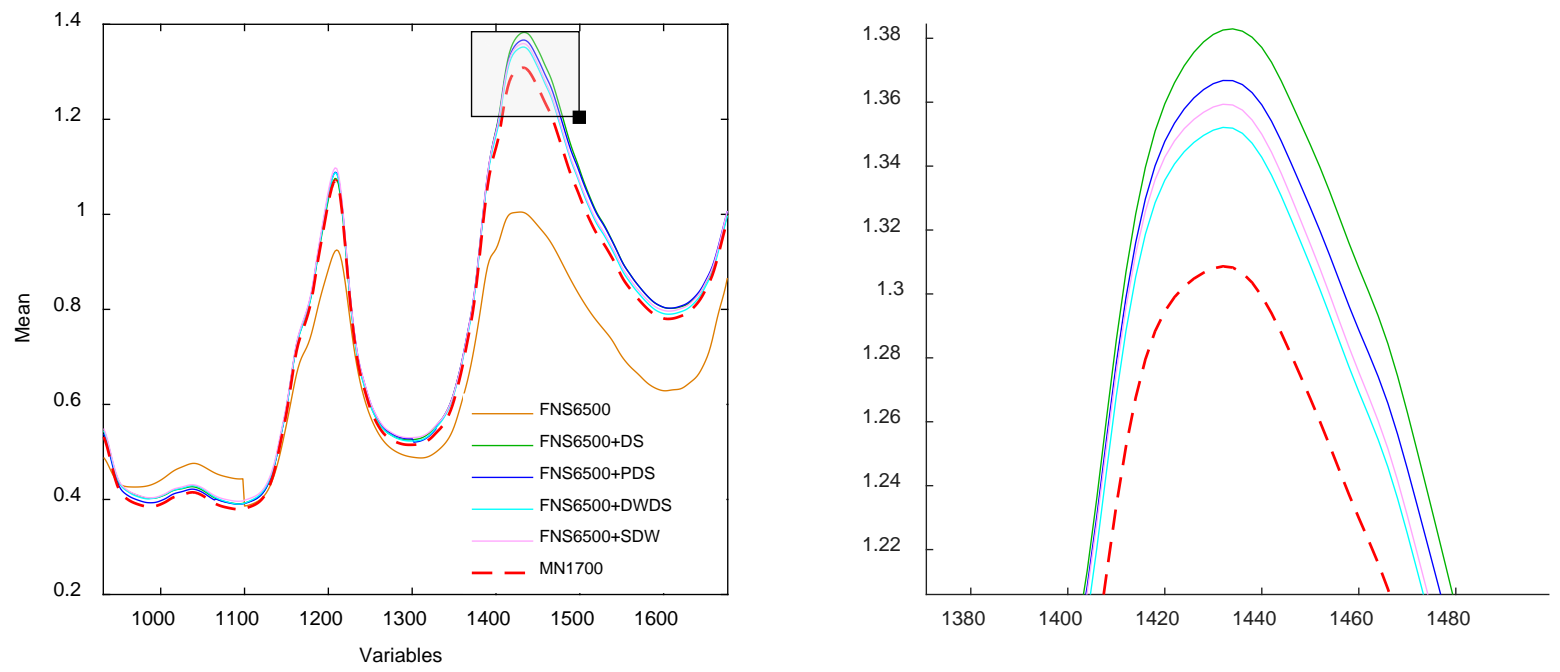

Figure 5. Average spectra (N=20) from FNS6500 before and after standardization and from MN1700. Right: detailed spectral area around $1450 \mathrm{~nm}$.

From a visual inspection of the spectra, before standardization, as expected, the FNS6500 spectrum, is far away of the MN1700 spectrum. However, when PDS, DWPDS and SDW are applied to the spectrum taken with the FNS6500, transferred average spectra are closer to the MN1700 average spectrum. It is important to notice that the spectral adjustment is not constant along the spectral range. Figure 5 shows that in the region of around $1200 \mathrm{~nm}$ which is a relevant wavelength for fat absorption, the fitting achieved by the different standardization algorithms is better than the obtained around $1400 \mathrm{~nm}$ which is characteristic of water absorption.

A Principal Component Analysis (PCA) was also developed in order to reduce the number of variables and represent a multivariate data table in a low-dimensional space. The distribution of all the samples along the first two principal components was examined (Figure 6). 

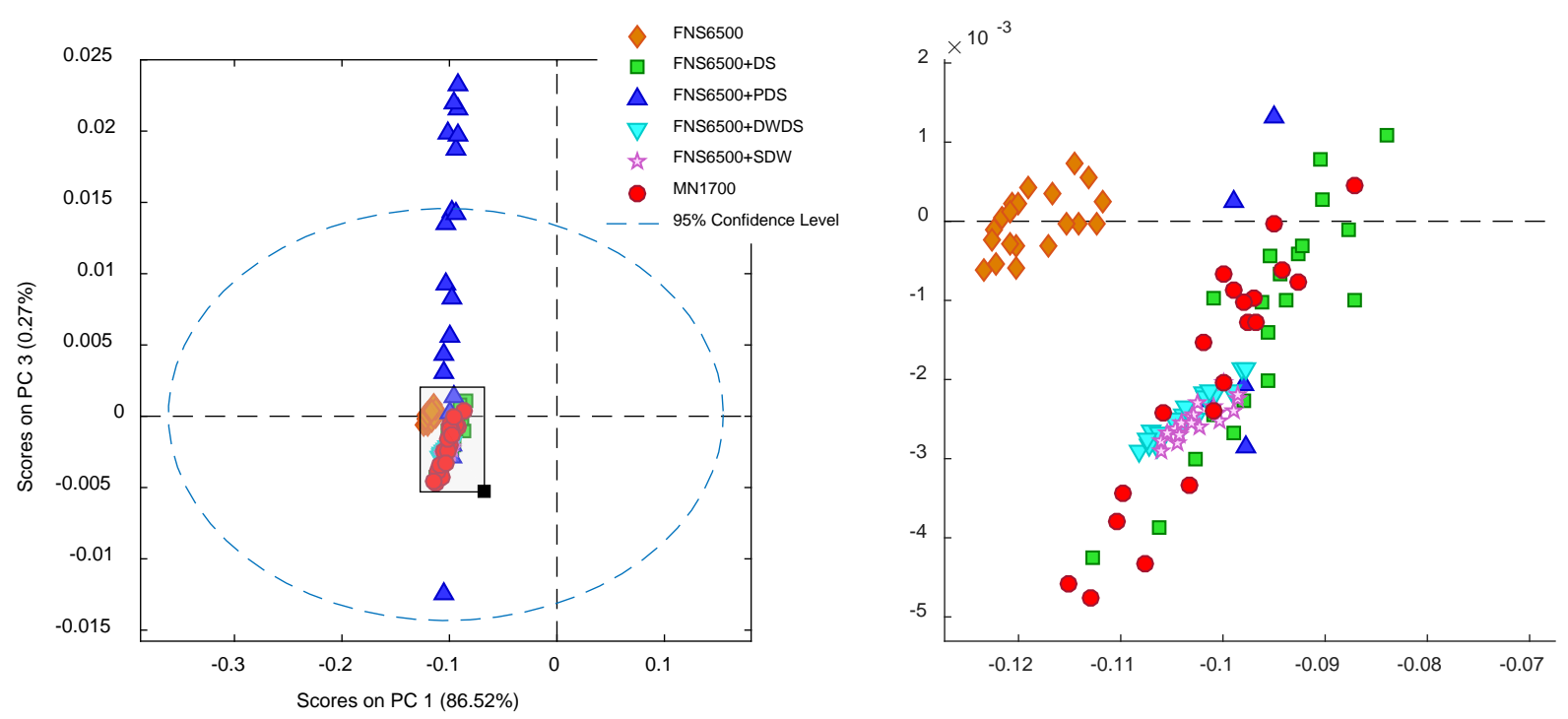

Figure 6. Projections into the first two principal components of spectra (N=470) from FNS6500 before and after standardization and from MN1700. Right: detailed PC area.

By analyzing the location of spectra into the first two principal components, transferred spectra using DS, DWPDS and SDW are lying nearby the spectra belonging to MN1700 instrument, while transferred spectra using PDS are completely separated from the rest of samples.

Thus, since from a visual inspection of transferred spectra, it was not clear which cloning strategy stands out among the rest by its proximity to MN1700 spectra, so the different standardized spectra were also evaluated by measuring their DD (Data Distance) and RMS(c) values from the MN1700 dataset. Results are shown in Table 3.

Table 3. RMS (c) and Data Distance (DD) values for the cloning validation set (N=20) analyzed on the FNS6500 and on the NM1700 before and after standardization using various cloning algorithms.

\begin{tabular}{lll}
\hline Standardization strategy & RMS (c) & DD (\%) \\
\hline MN1700 vs MN1700 & 17.646 & 3,35 \\
MN1700 vs FNS6500 & 108.457 & 18,27 \\
MN1700 vs DS & 30.536 & 6,31 \\
MN1700 vs PDS & 22.519 & 5,57 \\
MN1700 vs DWPDS & 24.679 & 5,33 \\
MN1700 vs SDW & 22.242 & 6,54 \\
\hline
\end{tabular}

The best spectral fit in terms of DD was obtained when applying the DWPDS algorithm, which yielded a fit of 5.33\%. The lowest RMS (c) were instead obtained using SDW cloning algorithm reaching a value of $22.242 \mu \log (1 / R)$, quite similar to the RMS(c) obtained between replicates in the MNS1700 (master instrument).

Other statistics providing information on spectral goodness of fit between instruments are the Mahalanobis global $\mathrm{H}$ (GH) and neighbor $\mathrm{H}(\mathrm{NH})$ distances. Spectral distances from the population center of the transferred calibration set $(\mathrm{N}=470)$ to the cloning validation set using the MN1700 dataset are shown in Table 4. 
Table 4. Mean GH values for the cloning validation set ( $\mathrm{N}=20$ samples).

\begin{tabular}{lll}
\hline Standardization strategy & GH & NH \\
\hline MN1700* vs FNS6500^ & 437.413 & 404.879 \\
MN1700* vs FNS650+DS^ & 2,75 & 0,30 \\
MN1700* vs FNS650+PDS^ & 2,06 & 0,36 \\
MN1700* vs FNS650+DWPDS^ & 2,96 & 0,47 \\
MN1700* vs FNS650+SDW^ & 3,43 & 0,75 \\
\hline
\end{tabular}

* val set $(\mathrm{N}=20)$

$\wedge$ cal set $(\mathrm{N}=470)$

The values recorded for the unstandardized FNS6500 validation set $(\mathrm{GH}=437.413$; $\mathrm{NH}=404.879)$ were widely higher than those recorded for the "FNS6500+standardization model" $(\mathrm{GH}=1,38 ; \mathrm{NH}=0,30)$ and exceeded the recommended cutoff limits of 3 and 0,6, respectively (Zamora et al. 2012b). After standardization, however, values for GH and NH lay within recommended limits in all cases but not in the FNS650+SDW, suggesting that, in general, the standardization considerably reduced the main spectral differences.

\section{CONCLUSIONS}

The most common inter-instrument standardization methods were presented and explained using spectra belonging to different instruments. Results obtained demonstrated the successful transferring of a large dataset of Iberian pig spectra analyzed in a monochromator lab instrument to a miniature low-cost and real-time miniaturized NIR instrument.

This finding is critical for the real implementation of NIRS at the IP and others pork industry at slaughterhouse level. Future work will be focused on deepening specific algorithms and other statistics for the evaluation of the spectral matching of the transferred spectra and the MN1700 spectra. New samples from the slaughter campaign 2017-2018 will be included into the MN1700 spectral samples database and models for the prediction of FAs and the discrimination between "Premium" and Non-premium" categories will be developed.

\section{ACKNOWLEDGMENTS}

The research leading to these results has received funding from the European Union's Seventh Framework Programme for research, technological development and demonstration under grant agreement No. 613688.

\section{REFERENCES}

De Boor, C., (1978). A Practical Guide to Splines, Springer-Verlag.

De Pedro, E., Garrido, A., Bares, I., Casillas, M., \& Murray, I. "Application of near infrared spectroscopy for quality control of Iberian pork industry”. In K.I. Hildrum, R. Isaksson, T. Naes \& A. Tandberg (Eds.). Near infrared spectroscopy bridging the gap between data analysis and NIR applications (pp. 345-348). UK: Ellis Horwood (1992).

De Pedro-Sanz, E., Serrano, A., Zamora-Rojas, E., Garrido-Varo, A., Guerrero-Ginel, J.E., Pérez-Marín, D., NúñezSánchez, N., y García-Casco, J.M. "Predicting Acorn-Grass Weight Gain Index using non-destructive Near Infrared Spectroscopy in order to classify iberian pig carcasses by feeding regime”. Grasas y Aceites, 64 (2), 210-218 (2013).

Fearn, T. 2001. "Standardisation and Calibration Transfer for near Infrared Instruments: A Review.” J. Near Infrared Spectrosc. 9, 229-244 (2001).

Fearn, T, Pérez-Marín D., Garrido-Varo A., Guerrero J.E. "Inverse, classical, empirical and nonparametric calibrations in a Bayesian framework.” Journal of NIRS 18 (1), 27-38 (2010). 
García-Olmo J.; De Pedro E. and Garrido-Varo, A. "Methodological aspects on near infrared analysis of Iberian pig fat using interactance-reflectance fiber optic mode”. Journal of NIRS. 6; p. 307-312 (1998).

García-Olmo, J., Garrido-Varo, A. and De Pedro, E. "The transfer of fatty acid calibration equations using four sets of unsealed liquid standardisation samples”. J. Near Infrared Spectrosc. 9, 49-62 (2001).

Garrido, A. \& De Pedro, E. “The role of near infrared spectroscopy in verifying label information in agro-forestry.” In: Handbook of near-infrared spectroscopy analysis. Practical spectroscopy series, Ed by D.A. Burns And E.W. Ciurczak, CRC Press, USA, 387-398 (2007).

Garrido-Varo A, García-Olmo J, Pérez-Marín D. “Analysis of Fats and Oils.” In C. Roberts, J.Workman and J. Reeves (Eds.) Near-Infrared Spectroscopy in Agriculture. Agronomy, Crop Science and Soil Science Societies of America, Madison, WI, USA, 487-558 (2004).

Garrido-Varo, A., D. Pérez-Marín, E. Zamora, E. De Pedro, J.E. Guerrero. „At-line and on-line NIRS analysis in the Iberian pig industry”. Invited paper International Difusse Reflectance Conference IDRC 2012, Chambersburg, PA (EEUU) (2012).

Hervás C., Garrido A., Lucena B., García N. y De Pedro E. "Near infrared spectroscopy for classification of Iberian pig carcasses using an artificial neural network”. J. Near Infrared Spectrosc. 2 pp. 177-184 (1994).

Pérez-Marín D., Garrido-Varo A., de Pedro E. y Guerrero-Ginel JE. "Chemometric utilities to achieve robustness in liquid NIR calibrations: application to pig fat analysis.” Chemom. Intell. Lab. Syst. 87(2); p. 241-246 (2007).

Perez-Marin, D.; Garrido-Varo, A.; Guerrero, J.E.; Fearn, T.; Davies, A.M.C. "Advanced Nonlinear Approaches for Predicting the Ingredient Composition in Compound Feedingstuffs by Near-Infrared Reflection Spectroscopy.” Applied Spectroscopy 62 (2008) 536-541 (2008).

Pérez-Marín D., Sánchez MT., Paz P., Guerrero JE., Garrido-Varo A., Soriano MA. Non destructive determination of quality parameters in nectarines during on tree ripening and postharvest storage. Postharvest Biology and Technology 52, 180-188 (2009).

Pérez-Marín D., Fearn T., Guerrero J.E., Garrido-Varo A. "Improving NIRS predictions of ingredient composition in compound feedingstuffs using Bayesian non-parametric calibrations.” Chemometrics and Intelligent Lab. Syst. 110 (1), $108-112\left(2012^{\mathrm{a}}\right)$.

Pérez-Marín, D.C., Fearn, T, Guerrero, J.E and Garrido-Varo, A. "Opportunities and Limitation of NIR Sensors for Massive Sampling”. Invited paper International Difusse Reflectance Conference IDRC 2012, Chambersburg, PA (EEUU) (2012b).

Shenk, J.S., \& Westerhaus, M.O. "Population structuring of near infrared spectra and modified partial least squares regression”. Crop Science, 31, 1548-1555. (1991).

Zamora-Rojas E., Pérez-Marín D., De Pedro E., Guerrero J.E., Garrido-Varo A. "In-situ Iberian pig carcass classification using a micro-electromechanical system (MEMS)-based near infrared (NIR) spectrometer". Meat Science 90 (3), 636-642 (2012a).

Zamora-Rojas, E.; Pérez-Marín, D.; De Pedro-Sanz, E.; Guerrero-Ginel, J.E.; Garrido-Varo, "A. Handheld NIRS analysis for routine meat quality control: Database transfer from at-line instruments.” Chemometrics Intell. Lab. Syst., 114, 30-512 35 (2012b).

Zamora-Rojas, E. "Control no destructivo e in situ de productos y procesos en la industria del cerdo ibérico usando sensores espectrales de infrarrojo cercano”. Thesis. Universidad de Córdoba. http://bit.ly/repositorio_tesis_EZR (2013).

Zamora-Rojas, E., Garrido-Varo, A., De Pedro-Sanz, E., Pérez-Marín, D., Guerrero-Ginel, J.E. Integración de sensores NIRS y tecnologías de la información y de la comunicación como motor de innovación en la industria del cerdo ibérico. ITEA Vol. Extra (2013). 